\title{
Personal Identification using quality image resulting from binarization and thinning techniques
}

\author{
T. Jyostna Rani \\ Assistant Professor, GIT, GITAM University, \\ Mahesh Kothuru \\ Assistant Professor, GIT, GITAM University,
}

\section{Abstract}

Biometric verification is any means by which a person can be uniquely identified by evaluating one or more distinguishing biological traits which include fingerprints, hand geometry, retina and iris patterns, voice recognition, signatures. Biometrics identification has eventually a much broader relevance as computer interface becomes more natural. It is mostly preferred mainly due to the invariance of the properties, measurability, singularity and acceptance. The oldest form of biometric verification is fingerprinting. It includes fingerprint registration and the fingerprint verification, where it goes through four phases which include binarization, thinning, minutiae extraction process and hough transformation. We use finger print verification based on fusion of minutiae and ridges using strength factors (FVMRSF), where this algorithm that has better matching percentage for different finger- prints compared to the existing algorithms and it has presentation based on the minutia Automatic Fingerprint Identification Systems.

\section{I.INTRODUCTION}

Electronic banking, e-commerce, and smartcards and an increased emphasis on the privacy and security of information stored in various databases, automatic personal identification has become a very important topic. Accurate automatic personal identification is now needed in a wide range of civilian applications involving the use of passports, cellular telephones, automatic teller machines, and driver licenses. Traditional knowledge-based (password or Personal Identification Number (PIN)) and token-based (passport, driver license, and ID card) identifications are prone to fraud because PINs may be forgotten or guessed by an imposter and the tokens may be lost or stolen. Therefore, traditional knowledge-based and token-based approaches are unable to satisfy the security requirements of our electronically interconnected information with the increasing emphasis on the automatic personal identification applications, biometrics especially fingerprint identification is the most reliable and widely accepted technique.

Fingerprints are today the biometric features most widely used for personal identification. Fingerprint recognition is one of the basic tasks of the Integrated Automated Fingerprint Identification Service (IAFIS) of the most famous police agencies. A fingerprint pattern is characterized by a set of ridgelines that often flow in parallel, but intersect and terminate at some points. Fingerprint biometric is the commonly used oldest and solely method internationally accepted as legal method to identify a person. Fingerprint ridges and valleys are unique and unalterable. Fingerprint biometric is used in numerous applications that include civilian and commercial applications like military, law enforcement, medicine, education, civil service, forensics, driver license registration, cellular phone 
access, computer log-in and like. Today live fingerprint readers based on optical, thermal, silicon, ultrasonic approach are used instead of old method of ink to capture FP.

FP identification is based on minutiae or location and direction of the ridge endings and bifurcations (splits) along a ridge path. The two commonly used .FP matching techniques are minutiae-based matching and pattern matching. Pattern matching just compares two image for checking similarity. Minutiae matching relies on minutiae points i.e. location and direction of each point. Experimental results show that the fingerprint based systems have very low FRR (False Rejection Rate) of 3 to $7 \%$.

By the below system architecture, we will be performing the operations as by the phases we have explained in the previous chapters regarding the pre-processing, feature extraction, feature selection, and fusion of the features which have been selected. Initially we will be taking a fingerprint of gray-scale image and then we will binarize the image such that we will have an image for the next pre-processing process to be ready for. Thinning will be done on the image to have the expansion of the valleys on the fingerprint and also for using this to reduce ridge width to one pixel. And then goes by the processes of filtering and final noise removal.

Now, we will be using the feature extraction techniques on the pre-processed image by minutiae extraction in the form of ellipse and removal of false minutiae if identified by any of the conditions mentioned before. Then, we will be using Hough transform where we will using it for the detection of the lines in the sample fingerprints we take for the experiment. Then, feature selection where we will be calculating the minutiae matching scores and ridge scores where in the final fusion, these two will be combined such that we will be calculating the final hybrid score for the particular fingerprint. In this way, we have completed executing our tasks for processing the fingerprint image.

\section{A. Project Definition}

In this project we are going to implement finger print verification based on fusion of minutiae and ridges using strength factors (FVMRSF).

In the preprocessing stage the Fingerprint is Binarised and Thinned. Next step is Minutiae Matching Score is determined using Block Filter and Ridge matching score is estimated using Hough Transform. Strength factors Alpha and Beta are used to calculate Hybrid Matching score.

\section{B. Problem Statement}

Fingerprint verification is most reliable characteristics for personal identification as it is unique and persistent. Fingerprint is also one of those features which does not change with the change of ages of the individuals. Therefore it is important to make sure that the fingerprint based systems to be as accurate as possible with the original fingerprints to be taken, so that the systems can be more efficient for the better purposes. In this report, we proposed Fingerprint Verification based on fusion of Minutiae and Ridges using Strength Factors in which the minutiae and ridge methods are combined. The Block Filter and Hough Transform are used to extract minutiae and ridges respectively. . The objective of this project is to get better matching percentage for different fingerprints using the FVMRSF algorithm compared to the existing algorithms. 


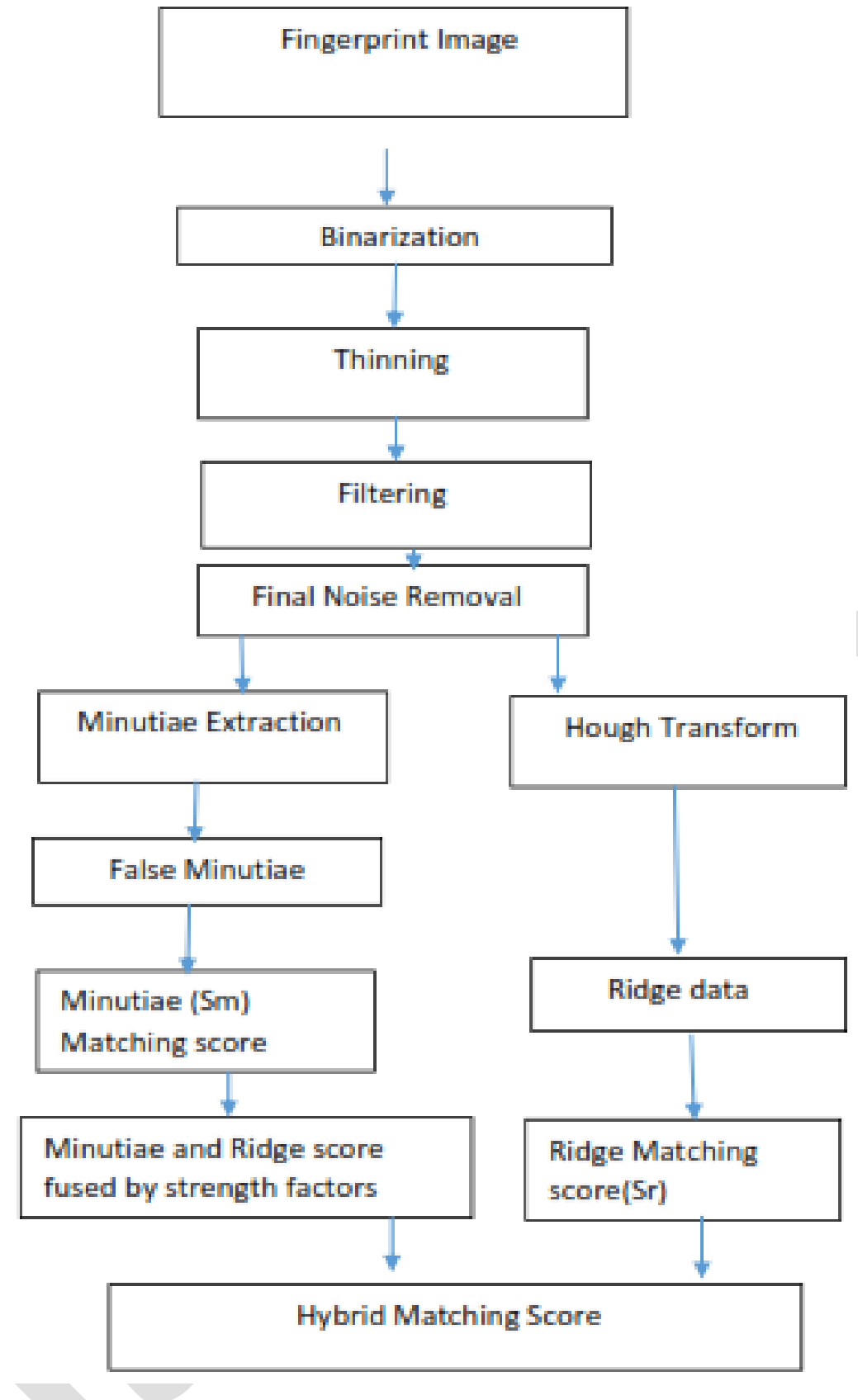

\section{FIGURE 1.1}

\section{LITERATURE REVIEW}

1. Michael Kucken and Newell discussed the hypothesis on the development of epidermal ridges. The epidermal ridge pattern is established as a result of buckling instability acting on the basal layer of the epidermis and resulting in the primary ridges. The buckling process underlying fingerprint development is controlled by the stresses formed in the basal layer and not by the curvatures of the skin surface and the stresses that determine ridge direction are themselves determined by boundary forces acting at creases and nail furrow, normal displacements which are most pronounced close to the ridge. Ashish Mishra proposed the algorithm based on orientation field of the fingerprint, over which gradient filter mask was used to detect the core points in the fingerprint 
image. Bazen and Gerez presented methods for the estimation of a high resolution directional field from fingerprints. The directional field detects the singular points and the orientations of the points. Yun and Cho proposed an adaptive preprocessing method, which extracts five features from the fingerprint images, analyzes image quality with clustering method, and enhances the images according to their characteristics. The preprocessing is performed after distinguishing the fingerprint image quality according to its characteristics.

2. Brankica M. Popovic and L. Maskovic used multiscale directional information obtained from orientation field image to filter the spurious minutiae. The feature extraction in pattern recognition system is to extract information from the input data and depends greatly on the quality of the images. Multiscale directional information estimated based on orientation field estimation. F.A.Afsar presented the minutiae based Automatic Fingerprint Identification Systems. The technique is based on the extraction of minutiae from the thinned, binarized and segmented version of a fingerprint image. The system uses fingerprint classification for indexing during fingerprint matching. G. Jagadeeswar Reddy presented fingerprint denoising using both wavelet and Curvelet Transforms. The search-rearrangement method performs better than minutiae based matching for fingerprint binary constraint graph matching since implicit alignment of two fingerprint images are not required. K. Zebbiche and F. Khelifi presented biometric images as one Region of Interest (ROI) that has the data processed by most biometric based systems. The scheme consists of embedding the watermark into ROI in fingerprint images. Discrete Wavelet Transform and Discrete Fourier Transform are used. Bhupesh Gour introduced midpoint ridge contour representation in order to extract the minutiae from fingerprint images. Colour coding scheme is used to scan each ridge only once. Seung Hoon chae and Jong $\mathrm{Ku}$ Kim proposed Fingerprint Verification in which both minutiae and ridge information are used to reduce the errors due to incomplete alignment or distortion.

3. Yi Chen and Anil K Jain proposed an algorithm based on fingerprint features viz., minutiae and ridges, Pattern and Pores. The correlation among Fingerprint features and their distributions are considered for the model. Johg Ku Kum described a study on of Hybrid fingerprint matching methods. The minutiae and image based fingerprints verification methods are implemented together. The shapes in the fingerprint such as square, diamond, cross and dispersed cross are used for matching. Manvjeeth Kaur proposed fingerprint verification system adopting many methods to build a minutiae extractor and a minutiae matcher. The method with some changes like segmentation using morphological operation, thinning, minutiae marking with special triple branch counting, Minutiae unification by decomposing a branch into three terminations, matching the unified $\mathrm{x}-\mathrm{y}$ co-ordinate system are employed. .Liu Wei described Rapid Singularity Searching for fingerprint classification. The algorithm uses Delta Poincare Index and Rapid Classification to classify the fingerprint into five classes. The Singularity is achieved by detection algorithm which searches the direction field that has the larger directional change. Arun Ross proposed the hybrid fingerprint matcher which employs the combination of ridge strengths and a set of minutiae points. Zhang Yuanyuan and Jing Xianjun presented the fingerprint image enhancement algorithm where the characteristics of the Gabor filter functions were analyzed both in spatial and spectral domain to obtain filter envelop parameters. These parameters were then convolved with the digital image to enhance the fingerprint image.

4. Anil K Jain described the use of logistic regression method to integrate multiple fingerprint matching algorithms. The integration of Hough transform matching, string distance matching and 2D dynamic programming based matching using the logistic regression has minimized the False Rejection Rate for a specified level of False Acceptance Ratio. Fanglin Chen proposed an algorithm for reconstructing fingerprint orientation field from saved minutiae and is used in the matching stage to compare with the minutiae from the query fingerprint. The orientation fields computed from the saved minutiae is a global feature and the saved minutiae are the local feature, are 
used to get more information. Chunxian Ren used the hybrid algorithm based on linear classifier to segregate foreground and background blocks. The pixel wise classifier uses three pixel features such as Coherence, mean and variance. Hartwig Fronthaler proposed a multigrid representation of a discrete differential scale space enhancement strategy of fingerprint recognition system. The fingerprint image is decomposed using Laplacian Pyramid as relevant information is concentrated within a few frequency bands.

5. Directional Filtering is used to enhance ridge valley pattern of fingerprint using 1-D filtering on higher pyramid level. The linear symmetric features are used to extract the local ridge valley orientation. Shabana Tadvi and Mahesh Kalte proposed the method of combining the minutiae features and wavelet statistical features to obtain the hybrid fingerprint matching. Liu Wei and Zhou Cong presented Gradual Segmentation algorithm and multi segmentation features for fingerprint image segmentation. The fingerprint region is obtained using a Gradual Segmentation and recoverable region is segmented using Multi Segmentation feature algorithm.

\section{A. Challenges}

Presentation based on the minutia Automatic Fingerprint Identification Systems. The technique is based on the extraction of minutiae from the thinned, binarized and segmented version of a fingerprint image. The system uses fingerprint classification for indexing during fingerprint matching. Introduction of midpoint ridge contour representation in order to extract the minutiae from fingerprint images. Color coding scheme is used to scan each ridge only once.

Described the use of logistic regression method to integrate multiple fingerprint matching algorithms. The integration of Hough transform matching, string distance matching and 2D dynamic programming based matching using the logistic regression has minimized the False Rejection Rate for a specified level of False Acceptance Ratio. Minutiae-based approaches are the most popular and widely used methods for fingerprint matching but minutiae are known to be unreliably extracted in low image quality conditions. For this and other reasons, alternative features have been proposed. The alternative feature most widely studied for fingerprint matching is texture information. The fingerprint structure consists of periodical repetitions of a pattern of ridges and valleys that can be characterized by its local orientation, frequency, symmetry, etc. Texture information is less discriminative than minutiae, but more reliable under low quality conditions.

From the above presented literature review papers and existing systems, we developed the problem statement for our proposed system for fingerprint recognition after going through series of papers which have been published based on the various fingerprint recognition techniques.

\section{B. Benefits}

a) Fingerprinting has high accuracy when used in comparing with other biometrics.

b) And, is the most economical biometric PC user authentication technique.

c) It is one of the most developed biometrics as it has been developed right from the earlier times.

d) Easy to use and can be as handy as possible.

e) Small storage space required for the biometric template, reducing the size of the database memory required.

f) It is standardized in almost in all the areas where the authentication is required.

Both of Sensor access control solutions can be integrated with fingerprint biometrics. When considering fingerprint biometrics it is important to consider the environment and the people who will be using them. If the environment or the user's hands are very dirty it would be worth considering readers which can read the finger a few layer of skin below the surface. For situations of where people do not want fingerprint data stored centrally, it would be worth considering storing their own fingerprint on a smart card that they carry with them. Using a smart card which carries your 
fingerprint means the reader itself no longer need to store the finger templates which means no limitation to its capacity. It's easy to see that the best biometric fingerprint authentication device offers many advantages and can be used effectively in many different applications. Although devices using this technology may require a larger investment in the beginning, they are more reasonably priced than ever.

\section{METHODOLOGY}

\section{A. FINGERPRINT PRE-PROCESSING}

Image pre-processing is the term for operations on images at the lowest level of abstraction. The aim of pre-processing techniques we are going to use is for an improvement of the fingerprint data we have collected so that it can suppress undesired distortions or enhances some image features relevant for further processing and analysis task. In our proposed method, the local pre-processing methods we have used are fingerprint extraction, binarization, thinning, filtering and final noise removal. The pre-processing methods we are going to implement, have been explained in the detailed way with the following methods in the following steps. Our process starts with the execution of the following steps:

\section{B. Finger- print image}

First, we will extract a gray- level fingerprint image (I) of size $640 * 480$ to perform the following techniques for image enhancement. A fingerprint in a narrow sense is an impression left by the friction ridges of a human finger. In a broader use of the term, fingerprints are the traces of an impression from the friction ridges of any part of a human. Fingerprinting is the reproduction of an epidermal layer of a finger that displays some typical characteristics, which are classified as valleys and ridges and can be analysed. First, we will extract a gray- level fingerprint image (I) of size $640 * 480$ to perform the following techniques for image enhancement. Extraction techniques must be used to obtain the fingerprint data. These techniques use binarization, thinning and features extraction algorithms which are computational methods.

\section{Binarization}

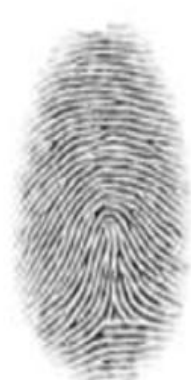

(a) Original image

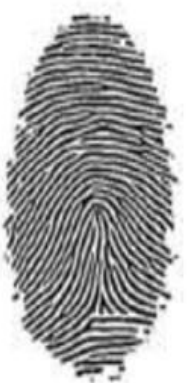

(b) Binarized image

The process of converting a grayscale image to binary image is known as binarization. In a gray-scale fingerprint image, a pixel can take on 256 different intensity levels. The threshold value is used to convert grayscale image to binary. The pixel values below the threshold are set to zero and the intensity values greater than the threshold is assigned one. In binary image the pixel values are assigned 0 and 1 to black and white pixels respectively. The processing of binary image is easy as it has only two intensity levels compared to gray scale image of 256 intensity levels. The diagram shows original fingerprint image and its corresponding binarized image. The disadvantage of binarization is that the ridges end near the boundary is considered as minutia even though it is not actual minutia. The problem of binarization is eliminated in thinning process.

\section{Thinning}


The thinning technique is often used to get the skeleton of an image. Thinning is a process of reducing the amount pixels in an image by removing all redundant pixels and producing a new simplified image with the minimum number of pixels possible, i.e successive application of two steps the points to points of the boundary region, and the contour pixel (P1) in the region is equal to 1 which has at least one 8-neighbor with a value 0.Generally, the image is covered by a mask, defined by $\mathrm{n}$ columns and $\mathrm{n}$ rows, in order to examine the values in the neighborhood of the central pixel 8neighborhood

From the previous step, we will notice that the image is in the better form after binarization. We will then be converting the thickness of ridge into single pixel wide by keeping original orientation and location of the minutiae to ensure accurate estimation by thinning. Now, we will use Block Filter to get a thinned image. Now, we will notice dilation in the valleys as a result the ridges are effectively eroded. Then, we will use a conservative structuring element consisting of four ones arranged in a two- by- two square which we will use it for the valley dilation to achieve some ridge width reduction, so that it can minimize the discontinuities.

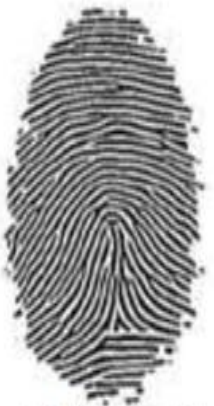

(a) Binarized image

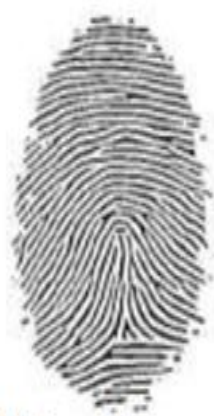

(b) Valley dilation

\section{E. Filtering}

As we did use the thinning method to preserve the outermost pixels along each ridge, the border is then fixed for fingerprint image to eliminate spur minutiae and we will then assign the pixel values as one for first five rows, last five rows, first five columns and last five columns. We will then perform the left to right scan for ridges that move up and right, and then we will perform the right to left scan for ridges that move down and left. Now we can be using this to reduce ridge width to one pixel. At last, we make sure that these two scans are combined to reduce ridge width to one pixel for whole fingerprint.
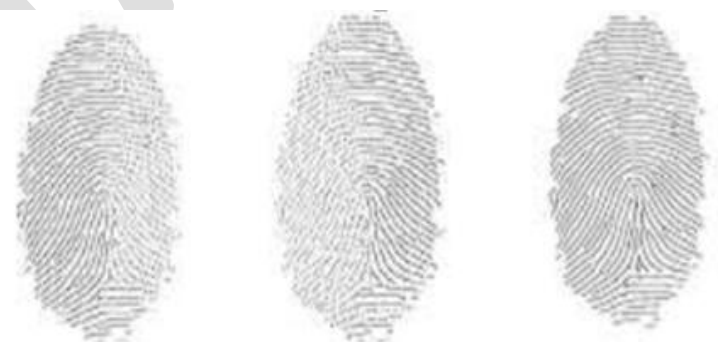

(a) Left to Right scan (b) Right to Left scan (c) Combined

In the above picture, we will notice the output fingerprint after both the scanning process have been performed and combined for the final result to get us a desired output. Wiener filter is used to filter out noise that has corrupted a signal. This technique assumes that if noise is present in the system, then it is considered to be additive white Gaussian noise. It is used in image restoration. Within the class of linear filters, the optimal filter for restoration in the presence of noise is given by wiener filtering. 


\section{F. Final noise removal}

If we notice any noise being produced by binarization and thinning, which we will eliminate by identifying the short island segments near the outer boundary of the image. Starting at each termination the ridge is traced pixel by pixel and if another termination is reached within the length of seventeen pixels from the termination, it is then considered as island segment and is deleted. If no termination is encountered until the maximum trace length is reached without any alterations, then we will make that segment to be retained.

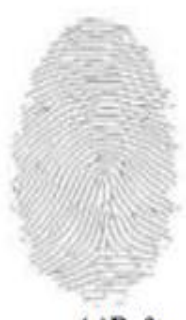

(a)Before

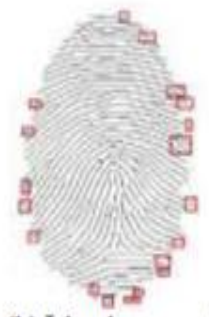

(b) Island segments to be deleted

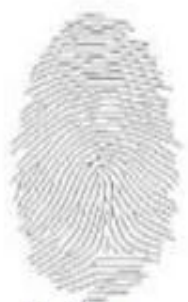

(c) After

Here, we will identify the island segments and then we will eliminate them to get the fingerprint without any further noise. However the fingerprint images contain noise caused by factors such as dirt grease, moisture and poor quality of input devices and storage devices. So in a noisy fingerprint image, ridges are not well defined and hence cannot be correctly detected. Noise might result in creation of false ridges and ignoring the genuine ridge lines Noise is the result of errors in the image acquisition process that result in pixel values that do not reflect the true intensities of the real scene. There are several ways that noise can be introduced into an image, depending on how the image is created.

\section{FEATURE EXTRACTION}

Feature extraction starts from an initial set of measured data and builds derived values intended to be informative and non-redundant, facilitating the subsequent learning and generalization steps as in our case to be in fingerprint recognition where we need minutiae extraction, false minutiae removal and ridge data using hough transform.

\section{A. Minutiae extraction:}

Minutiae points are the local ridge discontinuities, which are of two types: ridge endings and bifurcations. A good quality image has around 40 to 100 minutiae. It is these minutiae points which are used for determining uniqueness of a fingerprint. An accurate representation of the fingerprint image is critical to automatic fingerprint identification systems, because most deployed commercial large-scale systems are dependent on feature-based matching (correlation based techniques). Among all the fingerprint features, minutia point features with corresponding orientation maps are unique enough to discriminate amongst fingerprints robustly; the minutiae feature representation reduces the complex fingerprint recognition problem to a point pattern matching problem. In order to achieve high accuracy minutiae with varied quality fingerprint images, segmentation algorithm needs to separate foreground from noisy background which includes all ridge-valley regions and not the background. Image enhancement algorithm needs to keep the original ridge flow pattern without altering the singularity, join broken ridges, clean artifacts between pseudo-parallel ridges, and not introduce false information. Finally minutiae detection algorithm needs to locate efficiently and accurately the minutiae points. A number of binary image based methods are available which detect minutiae by inspecting the localized pixel patterns. They can be further classified into two classes, those that work on unthinned binarized images and those that work on thinned binarized images. 
Now, we make the fingerprint to undergo the minutia marking process such that the locations, where the ridges end at the outer boundaries of the image are marked as terminations. By creating an ellipse, we will eliminate the minutia outside an ellipse and retain the inside minutia. This method results in fast extraction of fingerprint minutiae that are based on the horizontal and vertical run length encoding from binary images without a computationally expensive thinning process.

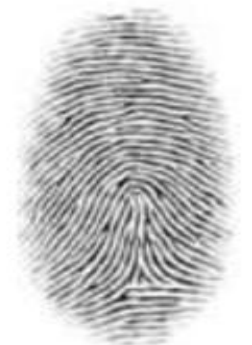

(a) Original image

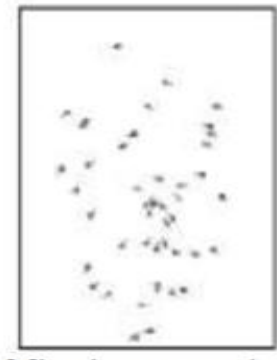

(b) Minutiae extracted

Minutiae detection can also be done directly from graylevel fingerprint images. A number of techniques exist, but it is still a topic of research. Extracting features directly from a gray scale image without binarization and thinning is of great relevance. Some fuzzy techniques have also been suggested in literature to extract minutiae from gray scale images directly. The approaches are distinguished on the basis of several factors like: the kind of input images they handle i.e. whether binary or gray scale, techniques of binarization and segmentation involved, whether thinning is required or not and the amount of effort required in the post processing stage, if exists. But low quality fingerprint images need pre-processing to increase contrast, and reduce different types of noises as noisy pixels also generate a lot of spurious minutiae as they also get enhanced during the pre-processing steps.

\section{B. False minutia removal:}

Now, we will take the average inter- ridge width (D) (i.e) the average distance between two neighboring ridges. The procedure in removing false minutia is:

(i) If the distance between one bifurcation and one termination is less than D and the two minutiae are in the same ridge, we will remove both of them.

(ii) If the distance between two bifurcations is less than D and they are in the same ridge, we will remove the two bifurcations.

(iii) If two terminations are within a distance $\mathrm{D}$ and their directions are coincident with a small angle variation, they satisfy the condition that no other termination is located between the two terminations, and then we will regard the two terminations as false minutia derived from a broken ridge, and we will remove them.

(iv) If two terminations are located in a short ridge with length less than $\mathrm{D}$, then we will remove the two terminations.

Due to noise in fingerprint images and imperfectness of the feature extraction methods, there are complex structures, such as loops and bridges, in the obtained ridge image. In order to make the ridge structures simple and consequently the matching algorithm easier, we will perform an operation, called clearup. (i) closed ridges are disconnected at an arbitrary point; (ii) ridges associated with bifurcations are split into three ridges; (iii) short ridges are removed.

\section{Ridge extraction using Hough transform:}

Now, we will use Hough transform to detect curves in images. Hough transform algorithm requires an accumulator array whose dimension corresponds to the number of parameters of the curve being detected. 


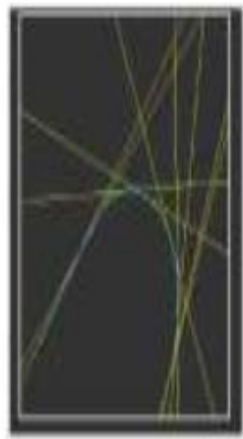

The above picture depicts the straight lines detected from a sample fingerprint ridge by using the Hough transform. The linear Hough transform algorithm uses a two-dimensional array, called an accumulator, to detect the existence of a line described by $r=x \cos \theta+y \sin \theta$. The final result of the linear Hough transform is a two-dimensional array (matrix) similar to the accumulator-one dimension of this matrix is the quantized angle $\theta$ and the other dimension is the quantized distance $r$. The well-known Generalized Hough Transform shows the superior performance in our proposed method. The most exciting properties of Hough transform are its immunity to noise. This approach converts point pattern matching to a problem of detecting peaks in the Hough space of transformation parameters. The best transformation parameters of matching two point sets corresponding the peak is attained by accumulating the evidence in the discretized Hough space.

\section{FEATURE SELECTION}

When the input data can be transformed into a reduced set of features so that the desired task can be performed by using this reduced representation instead of the complete initial data, this process is called feature selection. Here, for our project we have used the features, minutiae matching scores and ridge matching scores which we will get from the feature extraction techniques mentioned above.

\section{A. Minutiae Matching score:}

The process involves comparing the set of minutiae data extracted from input image to a set of minutiae data extracted from template image. The matching begins by creating a matrix, called rotate values, of the orientation angle difference between each template minutiae, $T k(1 \leq k \leq N T)$, and each input minutiae, $\operatorname{Im}(1 \leq m \leq N I)$.

$$
\left[\begin{array}{c}
r_{k}^{T} \\
\emptyset_{k}^{T} \\
\theta_{k}^{T}
\end{array}\right]=\left[\begin{array}{c}
\sqrt{\operatorname{row}_{k}^{T}}-\operatorname{row}_{r e f}^{T^{2}}+\operatorname{col}_{k}^{T}-\operatorname{col}_{k}^{T^{2}} \\
\tan ^{-1}\left(\operatorname{row}_{k}^{T}-\operatorname{row}_{r e f}^{T} \mid \operatorname{col}_{k}^{T}-\operatorname{col}_{r e f}^{T}\right) \\
\theta_{k}^{T}-\theta_{r e f}^{T}
\end{array}\right]
$$

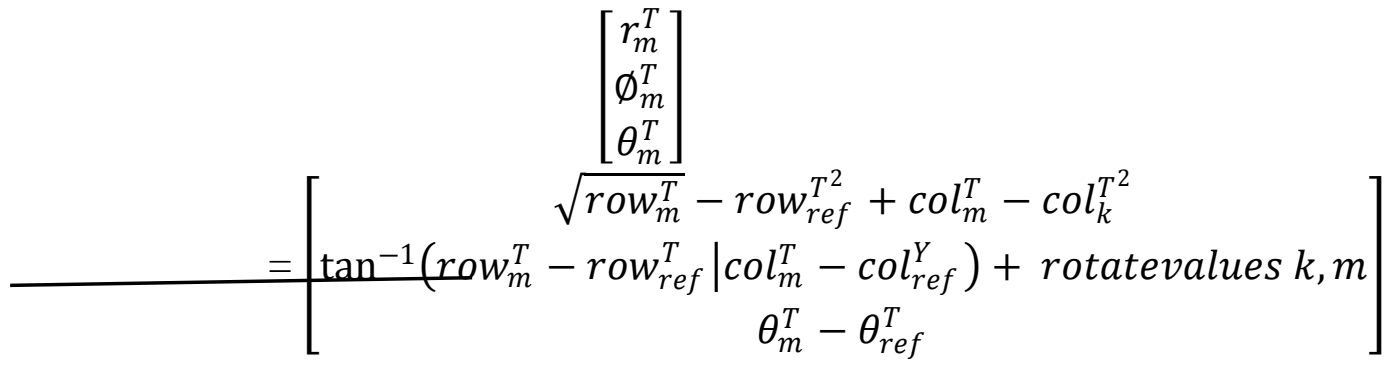

Minutiae matching score $(\mathrm{k}, \mathrm{m})=$ Number of minutiae pairs matched $/$ MAX $(\mathrm{Mi}, \mathrm{Mt})$

Where Mi and Mt represents the total number of minutiae in input image and total number of minutiae in template image 


\section{B. Ridge Matching Score}

Now we will find out the ridge matching score using the below formulae with the help of Hough Transform

Ridge Matching score $=$ Number of lines Matched in the image/Max $(\mathrm{Li}, \mathrm{Lt})$,

where Li and Lt Represents the total number of straight lines detected using Hough transform in an input image and total number of lines detected in the template image.

As two fingerprints may share a small overlapped region, in order to evaluate the similarity degree of them, we consider only the overlapped region. Compared with the bounding box of minutia points used in the convex hull of ridges is a more precise representation for border line of the fingerprints. In our implementation, the convex hull of ridges is approximated by the convex hull of sampled points of ridges. The overlapped region is the intersection of two convex hulls that are aligned using the initial substructure pair. We have proposed a fingerprint matching algorithm that matches both minutiae and ridges. A novel approach is used to find promising initial minutia pairs. For each initial minutiae pair, a ridge matching process is performed, which incrementally matches the remaining minutiae and ridges. Both minutiae and ridges are taken into account when computing the matching scores. Ridge image is an effective representation of the fingerprint image. From a ridge image, we can synthesize an image similar to the enhanced version of the original fingerprint image. On the contrary, it is definitely impossible to do so from a minutia set. Ridge image is also a compact representation of the fingerprint image. Ridge images can be efficiently approximated by polygonal lines, so the size of a template file is small.

\section{FUSION OF THE SCORES}

As we have obtained the ridge matching score and the minutiae matching score in the previous feature selection techniques, now we will be fusing both the scores with the help of strength factors.

\section{Minutiae and ridge score fused using strength factors}

Now, we will combine the minutiae and ridge matching scores are using following equations.

$\mathrm{HMS}=\alpha S r+\beta^{2} \mathrm{Sm}$

$\alpha+\beta=1$

Where

$\mathrm{Sr}=$ Ridge Matching Score

$\mathrm{Sm}=$ Minutiae Matching Score

HMS = Hybrid Matching Score

$\alpha$ and $\beta$ are strength factors

So, this method is mainly used by us in the aim to objectify that the matching percentage value of two different Fingerprints is low in this algorithm when compared to the existing algorithms for every value of $\alpha$ and $\beta$.

\section{ALGORITHM}

\section{A. Problem definition}

The Fingerprint is verified using Fingerprint Verification based on fusion of Minutiae and Ridges using Strength Factors.

a) Objectives of the algorithm are:

(i) The Minutiae Extraction using thinned image by Block Filter.

(ii) The true ridge information is extracted by Hough Transform.

(iii) The true minutiae and ridges information are combined to get matching score. 


\section{B. Algorithm of FVMRSF}

Input: Fingerprint Image

Output: Verified Fingerprint Image

1. Binarizing the gray scale fingerprint image.

2. Ridge thickness of binarized image is reduced to 1-pixel width by thinning of the image by Filtering.

3. Minutiae extraction is done on thinned image by considering the region of interest by using an Ellipse

4. False Minutiae removal.

5. Extracting the ridge information of the image by Hough Transform.

6. Compute the minutiae and ridge matching scores.

7. Compute the matching score.

\section{Fingerprint samples}

\section{RESULTS}

Below is the fingerprint sample from FVC2004, we have included in our project where we can see that the first fingerprint of each of the 16 sets of the fingerprint samples are the original fingerprints and the other fingerprints in the sets are the fingerprints which have been changed like in enlarged, modified, lightened, cut in few of the areas of the specific fingerprint. So then, in comparing with the first fingerprint of the set, we will calculate the percentages of ridge matching score, minutiae matching score, hough transformation score and hybrid matching score we derived from the previous scores. The threshold value we have considered to be is $65 \%$ hybrid matching score according to the algorithm we have proposed.

\section{CONCLUSION}

Fingerprint verification is most reliable characteristics for personal identification as it is unique and persistent. In this paper we proposed Fingerprint Verification based on fusion of Minutiae and Ridges using Strength Factors in which the minutiae and ridge methods are combined. The Block Filter and Hough Transform are used to extract minutiae and ridges respectively. The FVMRSF gives better results for different fingerprint matching compared to existing

\section{Sample 1}

\begin{tabular}{|l|l|l|l|}
\hline 1.1 & Ridge & Minutiae & FVMRSF \\
\hline 1.2 & 57.07 & 81.63 & 67.19 \\
\hline 1.3 & 58.05 & 73.64 & 67.82 \\
\hline 1.4 & 57.75 & 84.64 & 67.53 \\
\hline 1.5 & 57.24 & 87.64 & 67.36 \\
\hline
\end{tabular}




\begin{tabular}{|l|l|l|l|}
\hline 1.6 & 56.24 & 84.63 & 66.82 \\
\hline 1.7 & 57.16 & 68.65 & 67.26 \\
\hline 1.8 & 58.08 & 86.63 & 68.04 \\
\hline
\end{tabular}

algorithms. The tests are based on the following criteria: image quality resulting from binarization processes (qualitative), size of the fingerprint used in the algorithms of adaptive threshold location and a sufficient number of minutiae points. To obtain enough minutiae points, it is necessary to implement the thinning technique and process it after the binarized image with two algorithms.

\section{A. Future Scope}

In future, the Wavelet transform is introduced to improve the results further. However, fusion at the feature extraction (representation) level is expected to be more effective due to the richer source of information at this level. With the progress of time-frequency localization techniques, the robustness in face images based on signal processing techniques became comparable with statistical techniques. Spatial/frequency methods are suitable as there is variation in size, orientation and frequency of natural textures, Spatial/Spatial frequency methods are based on image representations that indicate frequency content in localized regions in the spatial domain. These methods overcome the shortcomings of the traditional Fourier based techniques. Such methods are able to achieve good localization in both the domains. They are consistent to human visual system theory. In this work, Wavelet based methods have been investigated.

\section{BIBLIOGRAPHY}

1. Manvjeet Kaur, Mukhwinder Singh, Akshay Giridhar and Parvinder S. Sandhu, "Fingerprint Verification System using Minutiae Extraction Technique," Proceedings of WorldAcademy of Science, Engineering and Technology, vol 36, pp 497-502, 2008

2. F. A. Afsar, M. Arif and M. Hussain, "Fingerprint Identification and Verification System using Minutiae Matching," National Conference on Emerging Technologies, pp.141-146, 2004.

3. G. Jagadeeswar Reddy, T. Jaya Chandra Prasad and M. N. Giri Prasad, "Fingerprint Image Denoising using Curvelet Transform," Proceedings of Asian Research Publishing Network Journal of Engineering and Applied Sciences, vol 3, no 3, pp. 31-35, June 2008.

4. Bhupesh Gour, T. K. Bandopadhyaya and Sudhir Sharma, "Fingerprint Feature Extraction using Midpoint Ridge Contour Method and Neural Network," Proceedings of International Journal of Computer Science and Network Security, vol.8, no.7, pp. 99-103, July 2008.

5. Shabana Tadvi and Mahesh Kalte, "A Hybrid System for Fingerprint Identification," International Journal of Computer Science and Engineering, vol 2, no 3, pp 767-771, 2010. 